\title{
Electron Correlation Effects in Double Photoionization of Earth Alkaline Atoms
}

\author{
Hany Hamdy \\ Atomic Physics Laboratory, Beni-Suef Faculty of Science, Cairo \\ University, Beni-Suef, Egypt.
}

The double photoionization (DPI) of calcium and strontium in the region of the $3 p \rightarrow 3 d$ and $4 p \rightarrow 4 d$ giant resonances at $31.41 \mathrm{eV}$ and $25.24 \mathrm{eV}$ respectively has been analyzed for equal and unequal energy sharing by the two emitted electrons. The study includes the case where the two electrons are ejected in two different angles with respect to the major axis of the linear polarization of the photon beam (the E-vector). Comparison is made with the available theoretical and experimental data for the triple differential cross section of helium.

\section{Introduction:}

Electronic states of atoms are often described by a single electronic configuration, which is antisymmetric combination of a single set of functions, called orbitals, each depending on the coordinate of only one electron, the so called a one electron model. The presence of electron-electron interaction makes the single electronic configuration only an approximation. Nevertheless this is a good approximation in most cases. Several theoretical methods exist that can accurately describe interelectron correlation in bound states. However, much less is known about electron correlation in continua, where the electrons are not bound to a nucleus. The lack of knowledge on interelectron correlation in the continua has stimulated studies of double photoionisation (DPI). This is a process that happens only due to interelectron correlation, and is particularly depend on the electron correlation in the continuum. In atoms other than $\mathrm{He}$ double photoionisation can occur in two limits. The first limit called direct double photoionization where the two electrons are emitted simultaneously. This is the subject of this paper, where a resonant state of the neutral atom is excited and then autoionizes into the continuum. The second limit is called twostep double photoionization. In this case the process proceeds through an intermediate state of the singly charged ion which decays by emitting an Auger electron. Atomic helium, where only direct double photoionization is possible, 
is the simplest system to investigate electron correlation. The interest in double photoionisation of He is enhanced by the fact that LS coupling can be assumed; therefore there is no resonant structure to complicate the process. For a recent review see Briggs and Schmidt (1) and references therein. The cross sections of double photoionization are differential with respect to the energies of the electrons and their direction of emission and are called triple differential cross sections (TDCS) and are parameterized by:

$$
\operatorname{TDCS}=\frac{\mathrm{d} \Omega}{\mathrm{dE}_{1} \mathrm{~d} \theta_{1} \mathrm{~d} \theta_{2}}
$$

In experimental studies of TDCS, the angular correlation between the two outgoing electrons is measured. This is made by measuring the variation in intensity of the count rate for one electron detected in coincidence with the other one as a function of the angle between them. If the two electrons are emitted in the plane perpendicular to the incident photon beam, the two angles of emission are denoted $\theta_{1}$ and $\theta_{2}$ and are normally measured relative to the major axis of linear polarization (E-vector) of the photon beam as shown in Fig. (1). Direct double photoionization of earth alkaline atoms; calcium and strontium is an excellent extension to the work on helium. At first one would expect a similar behavior to that of double photoionization of He. Within the single electronic configuration picture, the two outer most electrons that are ionized in $\mathrm{Ca}$ and $\mathrm{Sr}$ are also in an $\mathrm{S}^{2}$ shell around a closed subshell. Because initial and final states for velence double photoionization of $\mathrm{Ca}$ and $\mathrm{Sr}$ have the same symmetry as $\mathrm{He}$, the same selection rules apply.

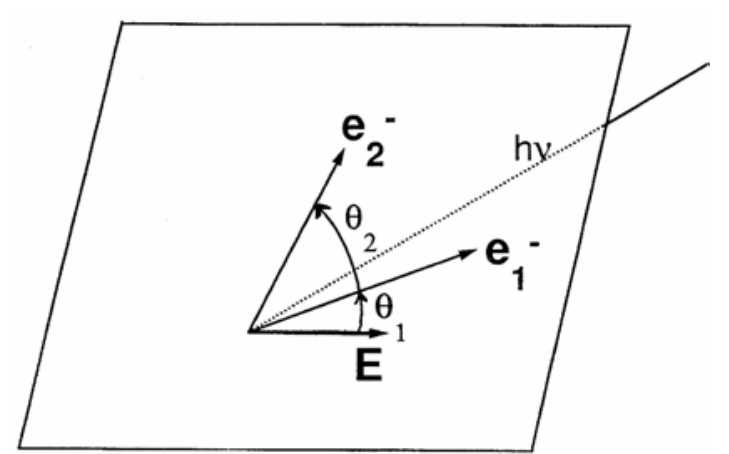

Fig. (1): The geometrical layout of the DFI process in the plane perpendicular to the incoming photon beam.

\section{Selection rules:}

Selection rules for differential DPI process have been obtained by Berakdar and Klar in 1992 (2) and by Maulbetsch and Briggs in 1995 (3) in the LS coupling scheme. These selection rules are valid for initial neutral and final doubly charged states both of ${ }^{1} \mathrm{~S}$ symmetry. These selection rules are applied for 
the measurements presented here; where the two electrons are detected in the plane perpendicular to the incident photon beam direction. The relevant selection rules predict that:

a) the TDCS is independent of the degree of linear polarization when the two electrons have the same kinetic energy (2).

b) Parallel and antiparallel emission of the two electrons is forbidden if the two electrons have the same kinetic energy ( 3 ).

c) emission of the two electrons, irrespective of their energies, in directions perpendicular to the linear polarization vector of the photon beam is forbidden (3).

\section{Coincidence Technique:}

In order to study direct double photoionization, the two specific electrons must be isolated from all other processes by observing them in coincidence. The word coincidence is used because the two electrons are emitted simultaneously or nearly so. For the measurements presented here, the DPI process of $\mathrm{Ca}$ and $\mathrm{Sr}$ proceeds through the following:

$$
\begin{gathered}
\mathrm{Ca} 3 \mathrm{p}^{6} 4 \mathrm{~s}^{2}{ }^{1} \mathrm{~S}_{0}+\mathrm{h} v_{\text {res. }} \longrightarrow \mathrm{Ca}^{*} 3 \mathrm{p}^{5} 3{\mathrm{~d} 4 \mathrm{~s}^{2}{ }^{1} \mathrm{P}_{1}}^{\longrightarrow} \mathrm{Ca}^{++} 3 \mathrm{p}^{6}{ }^{1} \mathrm{~S}_{0}+\overline{\mathrm{e}}_{1}+\overline{\mathrm{e}}_{2} \\
\mathrm{Sr} 4 \mathrm{p}^{6} 5 \mathrm{~s}^{2}{ }^{1} \mathrm{~S}_{0}+\mathrm{h} v_{\text {res. }} \longrightarrow \mathrm{Sr}^{*} 4 \mathrm{p}^{5} 4 \mathrm{~d} 5 \mathrm{~s}^{2}{ }^{1} \mathrm{P}_{1} \\
\mathrm{Sr}^{++} 4 \mathrm{p}^{6}{ }^{1} \mathrm{~S}_{0}+\overline{\mathrm{e}}_{1}+\overline{\mathrm{e}}_{2}
\end{gathered}
$$

The above process is a special case of direct DPI called resonant double photoionization in which the emission of the two valence electrons proceeds via a resonant state of the neutral atom, which then autoionize into the continuum.

For the measurements presented here the synchrotron radiation from the facility of Daresbury SRS was used as a source of the photon beam. The experimental apparatus and procedure are similar to those reported in reference (4). The two electrons are emitted with energies $E_{1}$ and $E_{2}$ or equivalently, $\mathrm{E}_{F}$ and $\mathrm{E}_{R}$ in the plane perpendicular to the photon beam at angles $\theta_{1}$ and $\theta_{2}$,or equivalently $\theta_{F}$ and $\theta_{R}$ respectively with respect to the direction of the major linear polarization; the E-vector. The indices $F$ and $R$ refer to the electrons accepted by the fixed analyzer and the rotatable analyzer respectively. 
One electron analyzer, $\mathrm{E}_{F}$ was fixed in position and it was set so that it accept electrons ejected either parallel to the E-vector of the incident radiation or perpendicular to it. Following Fig. (1), $\theta_{F}$ is the angle of the fixed analyzer $\mathrm{E}_{\mathrm{F}}$ and set either to $0^{\circ}$ or $270^{\circ}$. The second electron detector, $E_{R}$ rotated over a range of $180^{\circ}$ about an axis coincide with the incident radiation beam. In both cases for the fixed analyzer, the relative angle $\theta_{F R}$, or equivalently $\theta_{12}$, covers the range $90^{\circ}-270^{\circ}$.

\section{Total excess energy:}

In the present work measurements were made at a photon energy resonant with an excitation of the neutral atom in order to enhance the double photoionization process. The need to make measurements at resonant energy restricts the total excess energy $E_{\text {exc. }}$ to be shared by the two emitted electrons to the following:

$$
\mathrm{E}_{\text {exc. }}=\mathrm{E}_{F}+\mathrm{E}_{R}=\mathrm{h} v_{\text {res. }}-\operatorname{I} . \mathrm{P}\left(\mathrm{A}^{++}\right)
$$

Where letter A denotes to the neutral atom and I.P $\left(\mathrm{A}^{++}\right)$is the ionization potential of the doubly ionized atom. Double photoionisation of $\mathrm{Ca}$ and $\mathrm{Sr}$ takes place in the region of the giant resonance $\mathrm{np} \rightarrow \mathrm{nd}$ in $\mathrm{Ca}(\mathrm{n}=3)$ and $\mathrm{Sr}(\mathrm{n}=4)$ at $\mathrm{h} v_{\text {res. }}=31.41 \mathrm{eV}$ and $25.26 \mathrm{eV}$ respectively. A conservation of energy applied to equation (4) give values of $\mathrm{E}_{e x c}=13.43 \mathrm{eV}$ in case of $\mathrm{Ca}\left(\mathrm{I} . \mathrm{P}\right.$ of $\mathrm{Ca}^{++}=$ $17.98)$ and $\mathrm{E}_{\text {exc. }}=8.52 \mathrm{eV}$ in case of $\mathrm{Sr}\left(\mathrm{I} . \mathrm{P} \mathrm{Sr}^{++}=16.74 \mathrm{eV}\right)$.

\section{The resonance region $\mathrm{np} \rightarrow \mathrm{nd}:$}

Several resonances are present in the region of the $\mathrm{np} \rightarrow \mathrm{nd}$ excitation. The position of these resonances were found by recording a constant ionic state (CIS) spectra for the ns state of the singly ionized atom. Fig. $(2,3)$ show a CIS spectra for the $4 \mathrm{~s}$ and $5 \mathrm{~s}$ state of $\mathrm{Ca}^{+}$and $\mathrm{Sr}^{+}$respectively recorded at $\theta_{F}=0{ }^{\circ}$ and monochromator bandwidth of 100 and $150 \mathrm{~m} \mathrm{eV}$. The two figures indicate that, in addition to the main giant resonance at $31.41 \mathrm{eV}$ in $\mathrm{Ca}$ and at $25.24 \mathrm{eV}$ in $\mathrm{Sr}$, other resonances could be considered. However, the choice of these resonances would result in low coincidence rates and excessively long data acquisition time because of their weak intensity. 


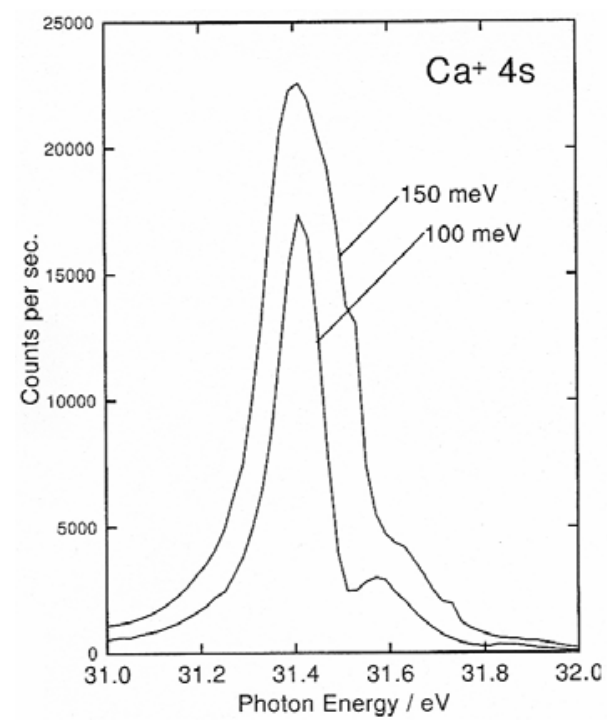

Fig. (2): CIS spectra of the $4 \mathrm{~s}$ state of $\mathrm{Ca}^{+}$in the region of the $3 \mathrm{p} \rightarrow$ $3 \mathrm{~d}$ resonance. Both spectra have been recorded with the fixed analyzer at $\theta_{F}=0^{\circ}$.

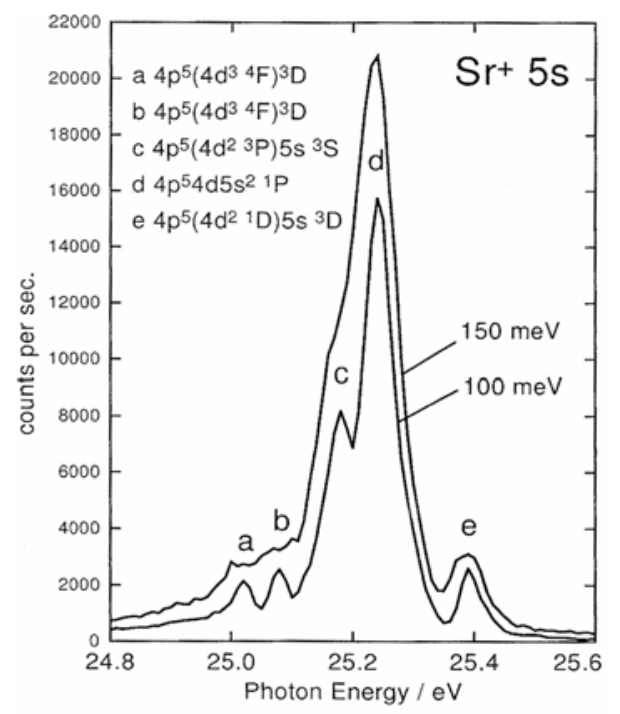

Fig. (3): As figure 2 but for the 5 s state of $\mathrm{Sr}^{+}$in the region of the $4 \mathrm{p} \rightarrow 4 \mathrm{~d}$ resonance. The structure has been assigned to the states identified by Mansfield and Ottley (19).

\section{Angular correlation measurements}

\subsection{Equal energy sharing}

Figures ( 4 \& 5) show the triple differential cross section, as defined in section (1), for DPI of Ca and Sr respectively. Data presented in Fig. (4 \& 5) are for equal kinetic energies of the two outgoing $S^{2}$ electrons measured at $\theta_{F}=0^{\circ}$. The cross-sections are presented in cartesian, for $\mathrm{Sr}$ data, and polar graphs, in case of $\mathrm{Ca}$ data, for later comparison with the corresponding measurements of He. Similar graphs could be drawn for the Sr data. The first experimental work on DPI of He was reported by Schwarzkopf et al (7) with $\mathrm{h} v=79 \mathrm{eV}$ for equal energy sharing of $\mathrm{E}_{1}=\mathrm{E}_{2}=10 \mathrm{eV}$. The two ejected electrons were analyzed in kinetic energy and direction of emission and detected in coincidence. The results for the symmetric energy sharing are show in Fig. (6). The TDCS of He was found to be in the shape of two symmetric lobes for antiparallel emission with a node at $\theta_{R F}=180^{\circ}$. Both Fig. (4 \& 5) show a deep minimum at $\theta_{R F}=180^{\circ}$, where in fact a zero value is expected from the selection rules outlined in section (2) and references $(2,3)$. This zero value is due to the unfavored character of the ${ }^{1} \mathrm{P}$ symmetry for the electron 
continuum. The fact that the minima at $\theta_{R F}=180^{\circ}$ are very deep can be taken as an indication that the present angular and energy resolution are sufficiently high to resolve all the angular structure in the TDCS. Symmetry about $\theta_{R F}=$ $180^{\circ}$ in these measurements must be expected because of the absence of circularly polarization of the incident photon beam (2). All available TDCS data for $\mathrm{He}$ and inert gases with the same experimental geometry as presented here show symmetry about $\theta_{R F}=180^{\circ}(2,5-8)$. However, data shown in Fig.(4) are not symmetric about $\theta_{R F}=180^{\circ}$. This asymmetry is believed to be an instrumental effect rather than a real property of TDCS. One possible reason for this is the slow change in the efficiency of the two analyzers with time; as the electron analyzers entrance slits are progressively coated with metal vapor. A another possible reason for this asymmetry may be a change in the overlap between the space "seen" by the two analyzers when one of them is rotated; although great care is taken to align the analyzers before measurements were taken. The asymmetry in the Sr data shown in Fig. (5) is not as strong as in Fig. (4) for Ca. In view of the comparison of the TDCS of $\mathrm{Ca}$ and $\mathrm{Sr}$ to the TDCS of He reported in reference (7) and represented her, Fig. (6), the shape of the Sr TDCS is closer to the single maximum structure (single pair of lobes) of $\mathrm{He}$ than is the case for Ca. The two pair of lobes structure in the TDCS of Ca is a reminiscent of the measured TDCS in the heavier rare gases $(5,6)$, where the DPI proceeds from the $3 \mathrm{p}^{6}$ shell. However, the lobes in the case of rare gases always extended outside the angular range $90^{\circ} \leq \theta_{R F} \leq 270^{\circ}$. The difference between the TDCS of $\mathrm{Ca}$ and that of $\mathrm{Sr}$ is very much in contrast to what would be expected on the basis that for $\mathrm{Sr}$, being heavier than $\mathrm{Ca}$, the configuration interactions are expected to be more important and TDCS with more than two lobes is expected. A possible reason for this difference could be a contamination from other resonant structure other than the main $3 \mathrm{p} \rightarrow 3 \mathrm{~d}$ resonance in $\mathrm{Ca}$, which could affect the Ca data. However, the measurements of Beyer et al (8) indicated that the on-resonant data were dominated by the main resonance peak. Moreover, the theoretical work on off-resonant DFI of Ca by Malegate et al (9) and by Kazansky and Obstrovsky (10) were both able to produce the four-lobe structure in TDCS of Ca. No reason is evident for the different number of lobes present in the TDCS of Sr compared to that of Ca. At this point it would be important to pursue a systematic study of how different resonances affect the TDCS. This is achieved partly by the works of Beyer et al (8), West et al (4) and Ross et al (11). The TDCS of $\mathrm{Ca}$ in the direct non-resonant continuum for equal energy sharing of the ejected electrons measured by Beyer et al (8) showed a change in the TDCS when moving from $\mathrm{h} v=31.41 \mathrm{eV}$ to $\mathrm{h} v=31.56$ $\mathrm{eV}$, which corresponds to a different resonance. The TDCS changes considerably and clearly confirm that TDCS is dependent on the resonance 
selected. The same conclusion is confirmed by West et al (4) for the TDCS of $\mathrm{Sr}$ on the low-energy side of the $4 \mathrm{p} \rightarrow 4 \mathrm{~d}$ resonance at $25.19 \mathrm{eV}$, where the shape of the angular dependence is different from that at the main resonance.

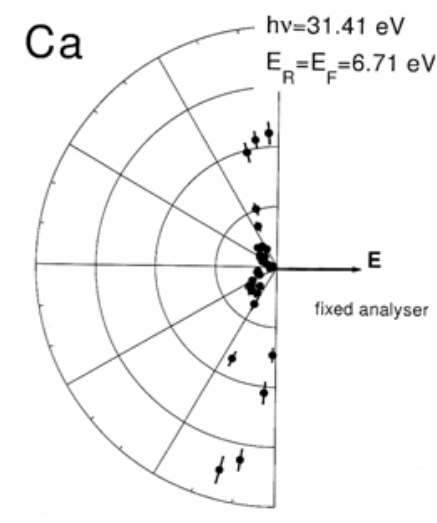

Fig. (4): TDCS of Ca for equal energy sharing of the two ejected electrons at the resonant photon energy of $31.41 \mathrm{eV}$, with angle $\theta_{F}$ set to $0^{\circ}$.

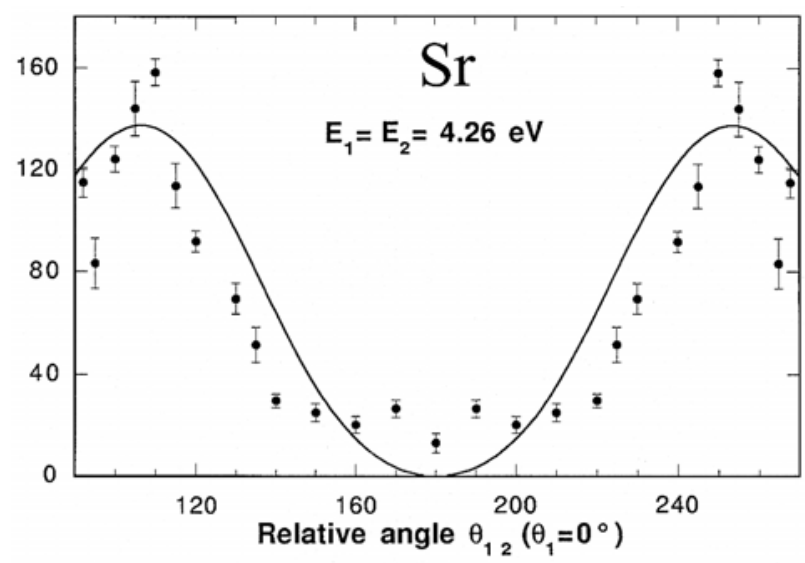

Fig. (5): As figure 4 but for $\mathrm{Sr}$ at the resonant photon energy of $24.25 \mathrm{eV}$, with angle $\theta_{1}\left(\theta_{F}\right)$ set to $0^{\circ}$. The continuous line is a fit to the data using equation 5 in reference ( 4 ).

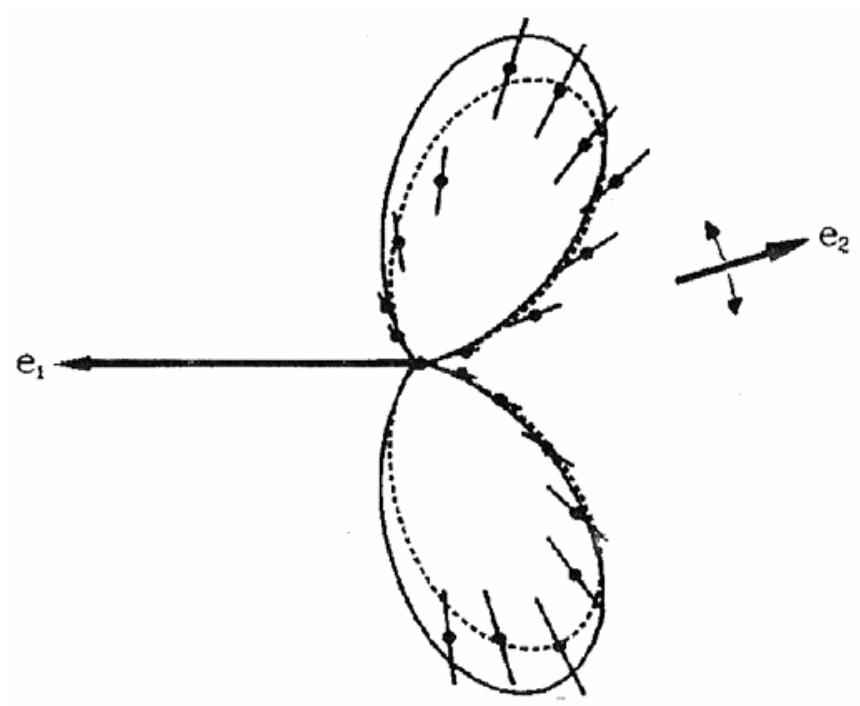

Fig. (6): TDCS for DFI of He at photon energy of $79 \mathrm{eV}$ and equal energy sharing of $10 \mathrm{eV}$ for the two ejected electrons. 
Figures $(7 \& 8)$ show the angular correlation between the two ejected electrons when they are emitted in the plane perpendicular to the photon beam and the fixed analyzer $\mathrm{E}_{F}$ set at $\theta_{R F}=270^{\circ}\left(-90^{\circ}\right)$ with respect to the E-vector of the photon beam. Applying selection rule (c) in section (2) a zero value for the TDCS is expected at $\theta_{R F}=180^{\circ}$. A nearly zero value is seen only in the case of Ca. The data for TDCS of Ca with the fixed analyzer set at $\theta_{F}=270^{\circ}$ are quite different from those at $\theta_{F}=0^{\circ}$. Instead of one strong pair of peaks at $100^{\circ}$ and $150^{\circ}$ and a weaker pair at $135^{\circ}$ and $220^{\circ}$, the low weak pairs are now of almost the same magnitude as the strong ones. The TDCS of Sr presented in Fig. (8) show a large cross-section at $\theta_{R F}=180^{\circ}$. West et al (4) measurements for the TDCS of Sr using much smaller angular increments $\left(2^{\circ}\right)$ than shown in Fig. (8) suggest that there might be (relaying on one point data only) a minimum at $\theta_{R F}=180^{\circ}$ and the central peak in the TDCS shown in Fig. (8) is in fact two peaks either side of $\theta_{R F}=180^{\circ}$ which can not be resolved with the angular resolution of $10^{\circ}$ of Fig. (8). Based on a theoretical work by Végh and Macek (11) for two-step DFI where the two ejected photoelectron and the Auger electron have the same energy and on the experimental work of Ueda et al (12) and West et al (4), the large cross section at $\theta_{R F}=180^{\circ}$ is most probably due to a substantial component of ${ }^{3} \mathrm{P}^{0}$ symmetry which contaminates the main $4 \mathrm{p} \rightarrow 4 \mathrm{~d}$ resonance.

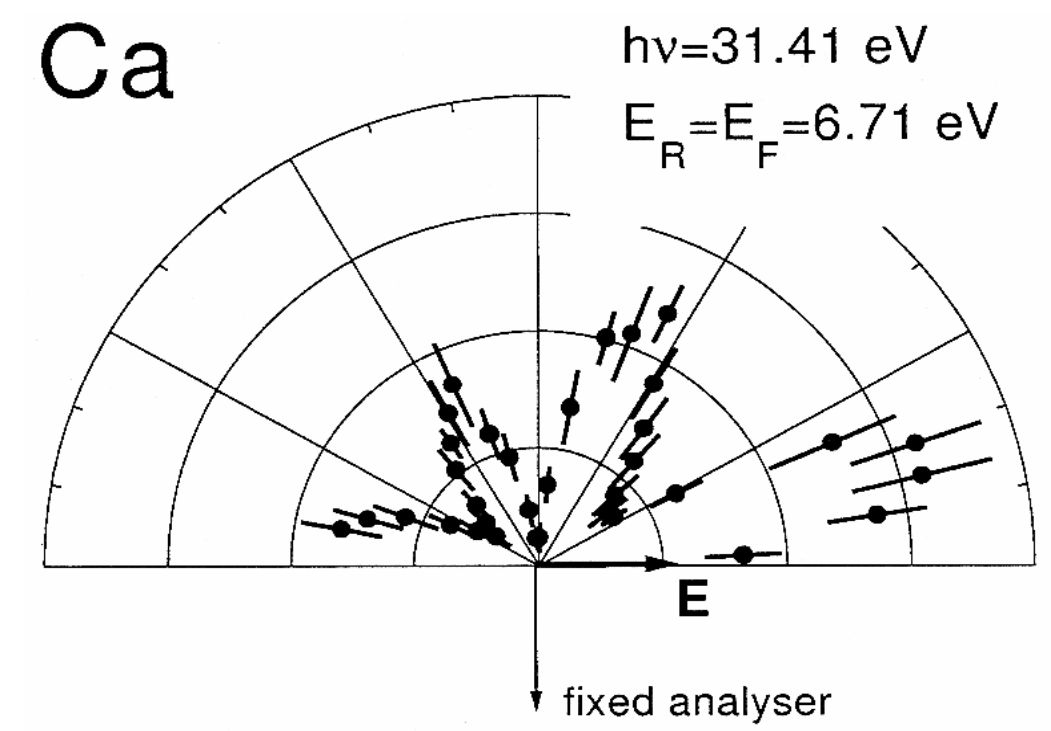

Fig. (7): TDCS of $\mathrm{Ca}$ for equal energy sharing recorded at resonant photon energy of $31.41 \mathrm{eV}$, with angle $\theta_{F}\left(\theta_{1}\right)$ set to $270^{\circ}\left(-90^{\circ}\right)$. 


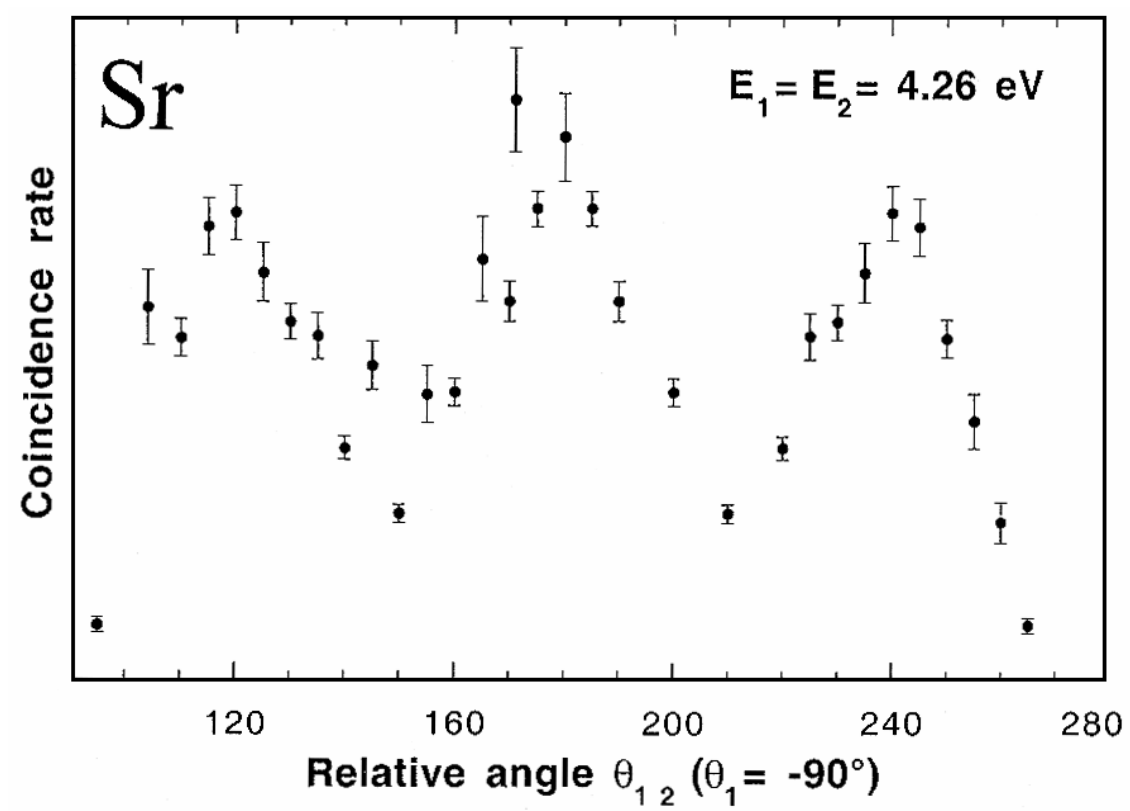

Fig. (8): As figure 7 but for $\mathrm{Sr}$ at the resonant photon energy of $24.25 \mathrm{eV}$ and angle $\theta_{1}\left(\theta_{F}\right)$ set to $-90^{\circ}\left(270^{\circ}\right)$.

\subsection{Unequal energy sharing:}

The data shown in Fig. $(9 \& 10)$ were made on the TDCS of $\mathrm{Ca}$ and $\mathrm{Sr}$ at the $\mathrm{np} \rightarrow \mathrm{nd}$ resonance at $31.41 \mathrm{eV}$ and $25.24 \mathrm{eV}$ respectively. These data were measured for unequal energy sharing and the fixed analyzer set at $\theta_{F}=0^{\circ}$. As pointed out in section (2), the selection rules forbid antiparallel emission of the two electrons when they have the same energy. When the electron energies are no longer equal, these selection rules break down: for nearly equal energies a minimum value for the TDCS should be present at $\theta_{R F}=180^{\circ}$, and for unequal energies this minimum gains in intensity and can become a maximum (13). Comparison between the graphs presented by Fig. $(4,5)$ for equal energy sharing and Fig. $(9,10)$ for unequal energy sharing show a clear peak at $\theta_{R F}=180^{\circ}$ for the unequal energy sharing case. This is in a good agreement with the selection rules where the symmetry of the equal energy case has been broken. The two shapes of the TDCS which correspond to the two cases of unequal energy sharing are expected to be complementary at angles other than $\theta_{R F}=180^{\circ}$. The two cases of the unequal energy sharing should be equivalent at $\theta_{R F}=180^{\circ}$. The overall profile of the TDCS of $\mathrm{Ca}$ shown in Fig. (9-a), is similar to that for equal energy sharing shown in Fig. (4) except for the non-zero cross section at $\theta_{R F}=180^{\circ}$. In the reverse situation presented in Fig. (9-b), there is clearly a second pair of maxima at $150^{\circ}$ and $200^{\circ}$ indicating 
that the TDCS for this condition depends strongly not only on the electron energy of the rotating analyzer but also on the energy of the electron in the fixed analyzer. This change of the TDCS shapes reflects the different influence of the slow electron on the fast one compared with the reverse situation. At this point, it is interesting to compare these TDCS shown in Fig. $(9,10)$ with the systematic study on the dependence of TDCS of He on energy sharing of $20 \mathrm{eV}$ reported by Brauning et al (14). In their work, a change from $\mathrm{E}_{\mathrm{F}}=15, \mathrm{E}_{\mathrm{R}}=5 \mathrm{eV}$ to $\mathrm{E}_{\mathrm{F}}=$ $5, \mathrm{E}_{\mathrm{R}}=15 \mathrm{eV}$ made a significant change in the TDCS of He. In the present work, for the TDCS of Sr, Fig. (10), a change from $E_{F}=1.76, E_{R}=6.76 \mathrm{eV}$ to $\mathrm{E}_{\mathrm{F}}=6.76, \mathrm{E}_{\mathrm{R}}=1.76 \mathrm{eV}$ does not have such a dramatic effect.

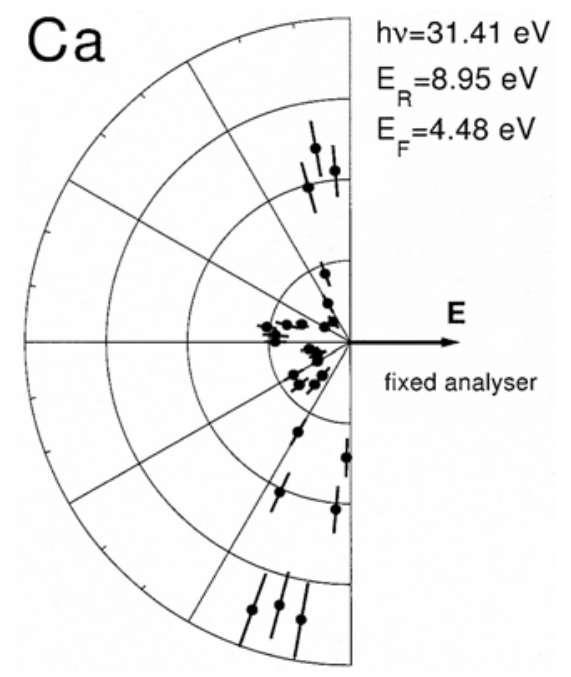

Fig.(9a):TDCS of $\mathrm{Ca}$ at the resonant photon energy of $31.41 \mathrm{eV}$ for unequal energy sharing of the ejected two electrons, recorded with angle $\theta_{F}\left(\theta_{l}\right)$ set to $0^{\circ}$. $\mathrm{E}_{\mathrm{F}}\left(\mathrm{E}_{1}\right)=4.48 \mathrm{eV}$ and $\mathrm{E}_{\mathrm{R}}\left(\mathrm{E}_{2}\right)=$ $8.95 \mathrm{eV}$.

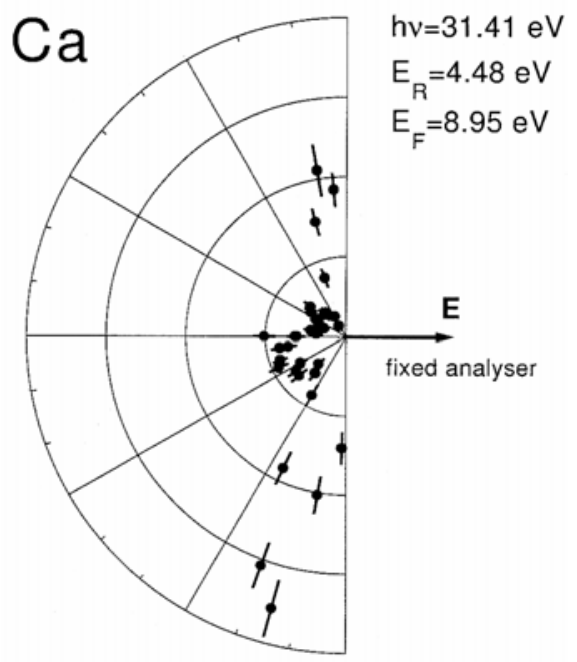

Fig.(9b): As figure 9-a but for $\mathrm{E}_{F}\left(\mathrm{E}_{1}\right)=8.95 \mathrm{eV}$ and $\mathrm{E}_{R}\left(\mathrm{E}_{2}\right)=4.48 \mathrm{eV}$.

Moreover, the TDCS of Sr showed stronger dependence on energy sharing than that of $\mathrm{Ca}$. The excess energy of $8.52 \mathrm{eV}$ in $\mathrm{Sr}$ compared to $13.43 \mathrm{eV}$ in $\mathrm{Ca}$ makes this result difficult to understand; as the dependence on energy sharing is expected to increase with the excess energy $(15,16)$. One reason that might be partly responsible for the greater change in TDCS of Sr when moving from equal to unequal energy sharing, compared to the TDCS of $\mathrm{Ca}$, is that the TDCS of Sr measured for unequal energy sharing where the difference in the energy of the two electrons is greater than that in the case of $\mathrm{Ca} ; 1.76$ and 6.76 for $\mathrm{Sr}, 4.48$ and $8.95 \mathrm{eV}$ for $\mathrm{Ca}$. However, it seems hard to believe that the difference in the dependence of TDCS on energy sharing can be entirely due to the different 
energies. It would be easier to believe that the difference between the dependence on energy sharing of the TDCS of $\mathrm{Ca}$ and $\mathrm{Sr}$ is a consequence of the different resonant states to which they are excited. In this context it would be interesting to measure TDCS for unequal energy sharing at one or more of the $\mathrm{Sr}$ and $\mathrm{Ca}$ resonances in the $\mathrm{np} \rightarrow$ nd region. These measurements are expected to be very challenging due to the weak intensity of the resonances other than the main $\mathrm{np} \rightarrow$ nd resonance, which make the coincidence data not useable on the Daresbury Synchrotron Radiation Source (SRS).

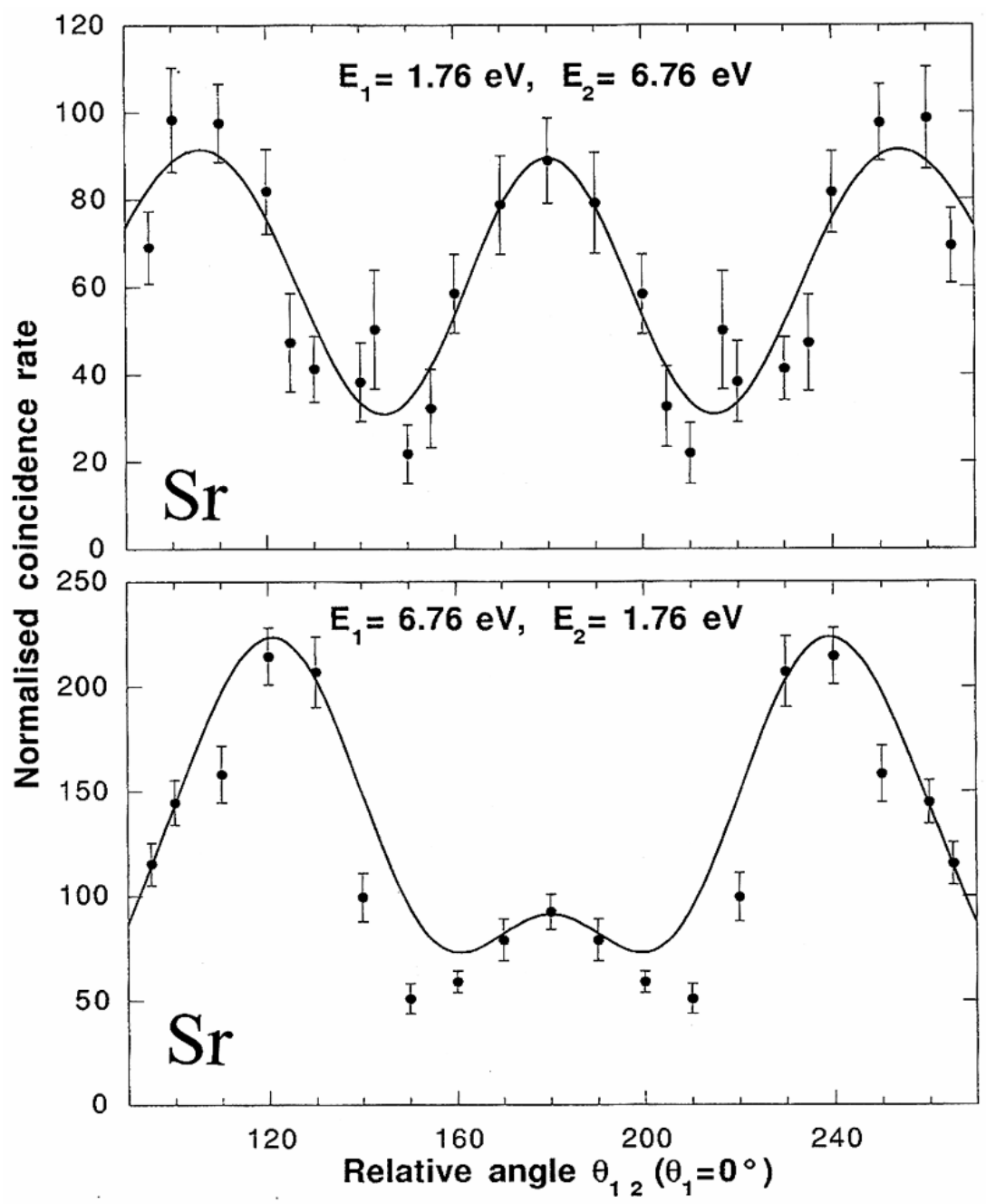

Fig. (10): As figure 5 but for unequal energy sharing of the two ejected electrons. Upper half for $E_{1}\left(E_{F}\right)=1.76 \mathrm{eV}$ and $E_{2}\left(E_{R}\right)=6.76 \mathrm{eV}$. Lower for $E_{1}\left(E_{F}\right)=$ $6.76 \mathrm{eV}$ and $\mathrm{E}_{2}\left(\mathrm{E}_{\mathrm{R}}\right)=1.76 \mathrm{eV}$. 
The TDCS of $\mathrm{Ca}$ and $\mathrm{Sr}$ measured for unequal energy sharing with the fixed analyzer set at $\theta_{F}=270^{\circ}$ are shown in Fig. $(11,12)$. As the two electrons are ejected perpendicular to the E-vector of the photon beam, the TDCS should be zero at $\theta_{R F}=180^{\circ}$ according to the selection rules. However, since the photon beam is not $100 \%$ plane polarized a minimum is expected at $\theta_{R F}=180^{\circ}$. A marked minima are presented in Fig. (11) for the TDCS of Ca. This is a distinct contrast to the case of the of Sr, Fig. (12), where a large cross section is evident at $\theta_{R F}=180^{\circ}$. In fact the TDCS of $\mathrm{Sr}$ at $\theta_{R F}=180^{\circ}$ is of similar magnitude to the two peaks at $120^{\circ}$ and $140^{\circ}$. Again as pointed out in section (6.1) this unexpected large cross section $\theta_{R F}=180^{\circ}$ for the TDCS of Sr could be due to a contamination of ${ }^{3} \mathrm{P}^{0}$ symmetry to the main $4 \mathrm{p} \rightarrow 4 \mathrm{~d}$ resonance where LS coupling was not found to be valid (12).

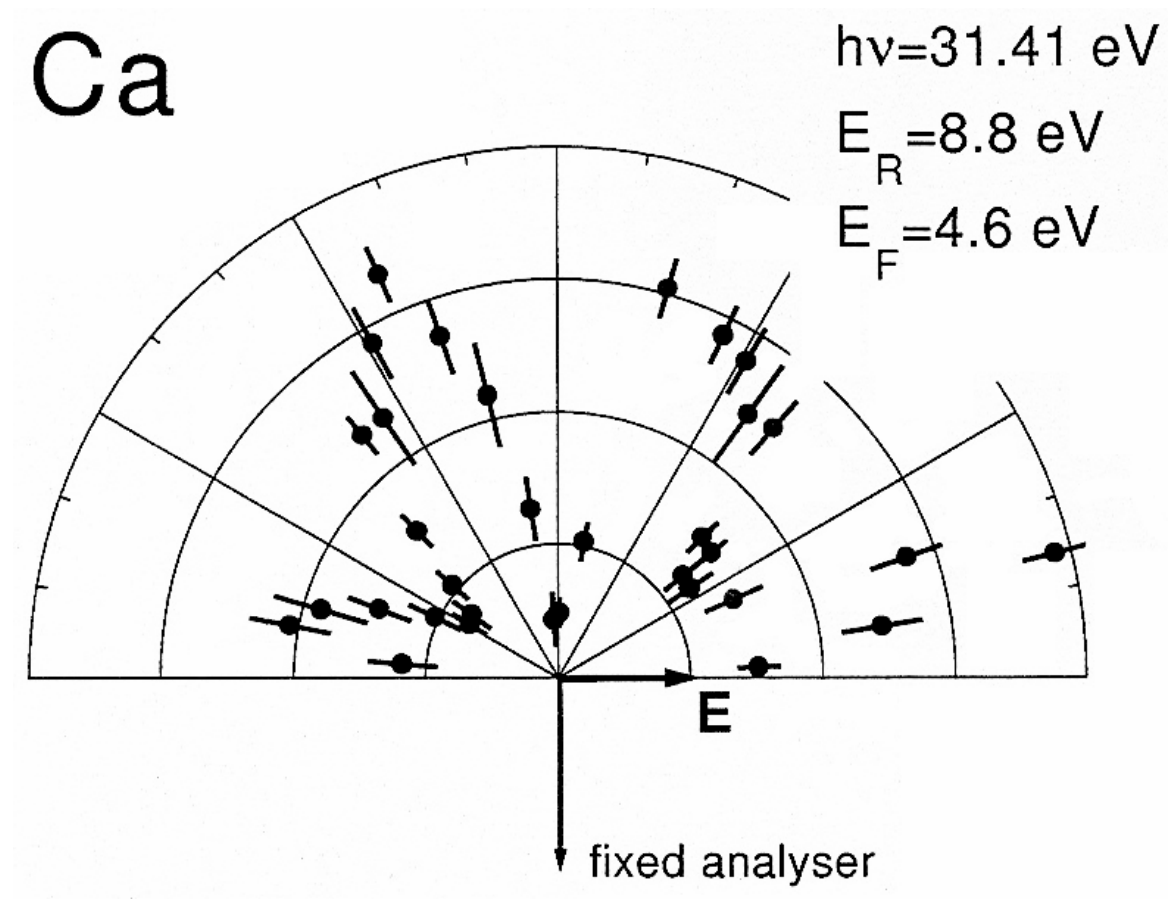

Fig. (11): As figure 7 but for unequal energy sharing of the two ejected electrons. 

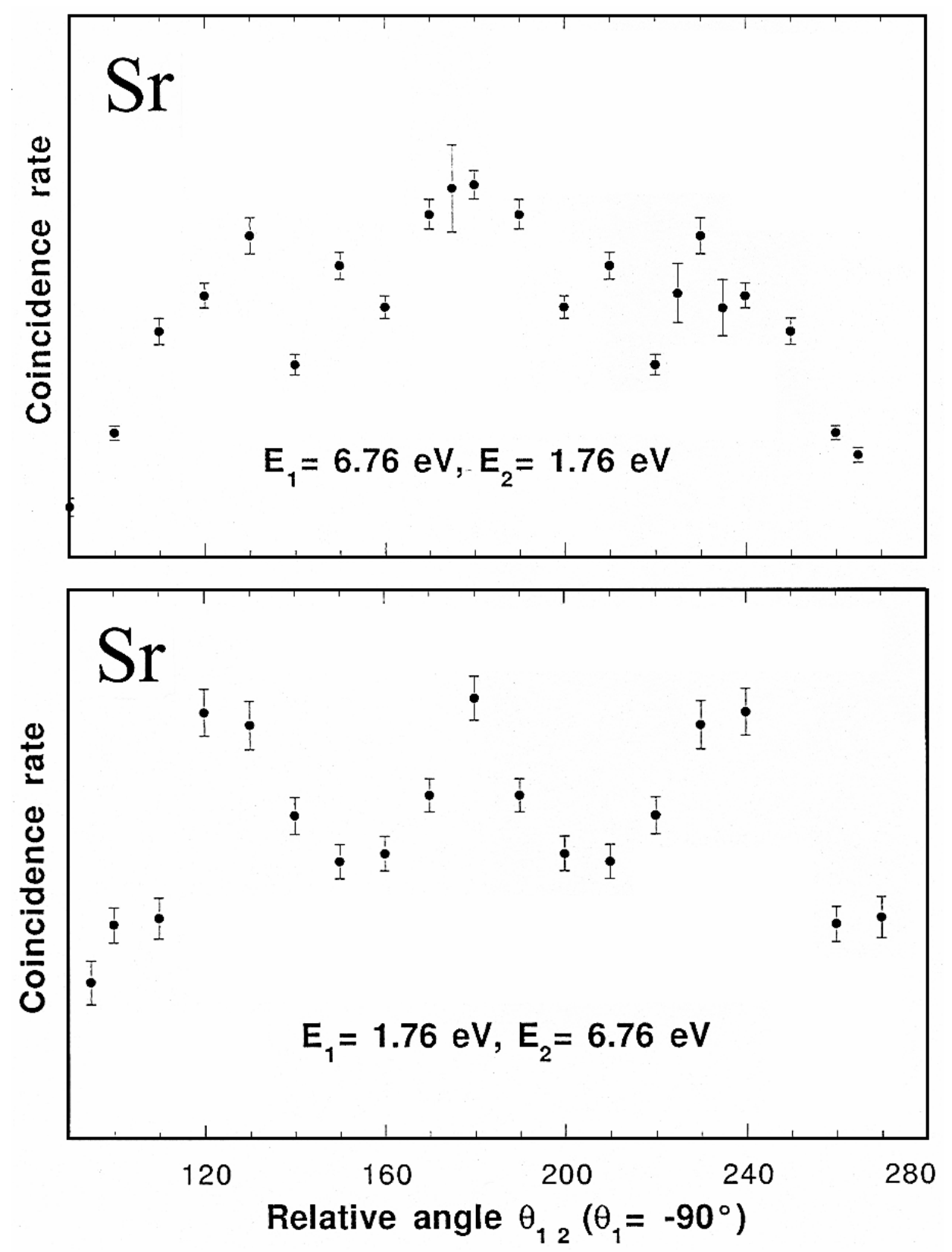

Fig. (12):As figure 8 but for unequal energy sharing of the two ejected electrons. Upper half for $\mathrm{E}_{1}\left(\mathrm{E}_{F}\right)=6.76 \mathrm{eV}$ and $\mathrm{E}_{2}\left(\mathrm{E}_{R}\right)=1.76$ $\mathrm{eV}$. Lower for $\mathrm{E}_{1}\left(\mathrm{E}_{F}\right)=1.76 \mathrm{eV}$ and $\mathrm{E}_{2}\left(\mathrm{E}_{R}\right)=6.76 \mathrm{eV}$. 


\section{Concluding Remarks:}

The dependence of the TDCS on the selected resonance confirm that the process under investigation here can not be regarded as pure double photoionization, but it must be seen as double autoionization of the excited neutral state to the continuum of the doubly charge atom. The calcium double photoionization in the region of the $3 p \rightarrow 3 d$ resonance has been shown to depend strongly on the direction in which the fixed angle electron is observed relative to the E-vector of the photon beam. Similar behavior was found in strontium except that a marked peak at $\theta_{R F}=180^{\circ}$ and $\theta_{F}=270^{\circ}$ appeared. The most likely explanation for this is that the $4 p \rightarrow 4 d$ resonance does not have pure ${ }^{1} \mathrm{P}^{0}$ symmetry. The calcium TDCS for unequal energy sharing by the two ejected electrons confirms that the TDCS for $\theta_{R F}=180^{\circ}$ and $\theta_{F}=270^{\circ}$ is nonzero. This is in contrast with the case of strontium under the same conditions. The study indicates the effect of the electron-electron correlation and/or the strong mixing between the resonance state and other doubly ionized continua. There are very few theoretical data on the DFI of metal atoms. However, it is hoped that the data on earthalkaline atoms act as a stimulus for theoretical calculations. At the same time, off-resonance measurements are required to enhance the theory of the double photoionization process.

\section{Acknowledgments:}

The author is grateful to Professor J B West and Professor K J Ross for financial support, in the form of travel grants, to carry out this work. I thank Dr. H-J Beyer and Dr. M Derbali for their help in preparing the original manuscript. I acknowledge the hospitality and help of the staff at Daresbury Laboratory, U.K

\section{References:}

1. JS. Briggs and V. Schmidt J. Phys. B: At. Mol. Opt. Phys. 33 R1 (2000)

2. J. Berakdar and H. Klar Phys. Rev. Letters, 69, 1175 (1992)

3. F. Maulbetsch and Briggs JS. J.Phys. B: At. Mol. Opt. Phys. 28 (1995)

4. West JB, Ross KJ, Beyer H-J, DeFanis A and Hamdy H. J.Phys.B: At. Mol. Opt. Phys. 34, 4169 (2001)

5. Krassig B, Schwarzkoph O and Schmidt V.J. Phys.B: At.Mol. Opt. Phys. 26, 2589 (1993)

6. SJ. Schaphorst, Jean A, Schwarzkopf O, Lablanqui P, Andric L, Huetz A, J Mazeau and V. Schmidt, J.Phys.B: At. Mol. Opt. Phys. 29, 1901 (1996)

7. O. Schwarzkopf and V. Schmidt, J.Phys.B: At. Mol. Opt. Phys. 28, 2847 (1995) 
8. O. Schwarzkopf, B. Krassig, J. Elmiger and V. Schmidt Phys. Rev. Letters, 70, 3008 (1993)

9. H-B. Beyer, JB. West, KJ. Ross and A. DeFanis J. Phys. B: At. Mol. Opt. Phys. 33, L767 (2000)

10. L. Melegate, F. Citrini, P. Selles and P. Archirel J. Phys.B: At. Mol. Opt. Phys. 33, 4209 (2000)

11. AK. Kazansky and VN. Ostorovsky J. Phys.B: At. Mol. Opt. Phys. 30, L835 (1997)

12. KJ. Ross, JB. West, H-J. Beyer and A. DeFanis J.Phys. B: At. Mol. Opt. Phys. 32, 2927 (1999)

13. L. Végh and JH. Macek Phy. Rev. A50, 4031 (1994)

14. K. Ueda, JB. West, KJ. Ross, H-J. Beyer and NM. Kabachnik J.Phys.B: At. Mol. Opt. Phys. 31, 480 (1998)

15. O. Schwarzkopf, B. Krassig, V. Schmidt, F. Maulbetsch and JS. Briggs J.Phys. B: At. Mol. Opt. Phys. 27, L347 (1994)

16. H. Bräuning, R. Dörner, CL. Coke, MH. Prior, B. Krassing, AS. Kheifets, I. Bray, A. Brauning-Demian, K. Carnes, S. Dreuil, V. Mergel, P. Richard, J. Ulrich and H. Schmidt-Bocking, J.Phys.B: At. Mol. Opt. Phys. 31, 5149 (1998)

17. A. Huetz, P. Selles, D. Waymel, and J. Mazeau, J.Phys.B: At. Mol. Opt. Phys. 24, 1917 (1991)

18. G. Dawber, L. Avaldi, AG. McConky, H. Rojas, MA. MacDonald and GC. King, J.Phys.B: At. Mol. Opt. Phys. 28, L271 (1995)

19. BWD. Mansfield and TW. Ottley, Proceeding of the Royal Society of London, 377, 431 (1981) 